\title{
INVESTIGACIONES
}

\section{Prácticas inclusivas del profesorado en aulas de escuelas chilenas: Un estudio comparativo}

\author{
Inclusive practices of teachers in classrooms of Chilean schools: \\ A comparative study
}

\author{
Laura Espinoza $a^{a, b}$, Karen Hernández ${ }^{c}$, Diana Ledezma ${ }^{c}$ \\ ${ }^{a}$ Departamento de Educación, Universidad de Los Lagos. \\ laura.espinoza@ulagos.cl \\ ${ }^{b}$ Colaboradora Instituto Interuniversitario de Investigación Educativa IESED. \\ ${ }^{c}$ Universidad Santo Tomás, Sede La Serena. \\ karenadriana.hernandez@alumnos.santotomas.cl, dianabelen.ledezma@alumnos.santotomas.cl
}

\begin{abstract}
RESUMEN
La existencia de aulas diversas en las escuelas de educación regular actualmente significa un desafío para los docentes en ejercicio en Chile. Dicha situación demanda que el profesorado implemente una enseñanza ajustada a las características del estudiantado. Este estudio tiene como objetivo identificar y comparar las prácticas pedagógicas inclusivas que el profesorado declara ejecutar en dos colegios de la Región de Coquimbo, Chile. La investigación se efectuó desde el enfoque cuantitativo, considerando 88 docentes participantes de diversa formación pedagógica y años de experiencia. Se recogió información por medio del instrumento Guía de Evaluación de Prácticas Inclusivas en el Aula GEPIA. Se hallaron diferencias en algunas prácticas pedagógicas de aula, sobre todo considerando el sexo y la formación de base docente. Los hallazgos son relevantes, pues generan un panorama de las distintas prácticas pedagógicas implementadas, y sobre cuáles los docentes dicen efectuar mayores ajustes para dar respuesta educativa a la diversidad.
\end{abstract}

Palabras claves: Prácticas pedagógicas inclusivas, inclusión educativa, formación docente, metodologías, evaluación.

\section{ABSTRACT}

The existence of diverse classrooms in regular education schools currently represents a challenge for practicing teachers in Chile. This situation demands that the teaching staff implement a teaching adapted to the characteristics of the student body. The goal of this study is to identify and compare the inclusive pedagogical practices that teachers declare to execute in two schools in the Region of Coquimbo, Chile. The investigation was carried out from the quantitative approach, considering 88 participating teachers of diverse pedagogical training and years of experience. Information was collected through the instrument Guide for the Evaluation of Inclusive Practices in the GEPIA Classroom. Differences were found in some classroom pedagogical practices, especially considering sex and teacher-based training. The findings are relevant, generating an overview of the different pedagogical practices implemented, and on which the teachers say to make greater adjustments to provide an educational response to diversity.

Key words: Inclusive pedagogical practices, educational inclusion, teacher training, methodologies, evaluation. 


\section{INTRODUCCIÓN}

Actualmente en Chile se observa que los establecimientos de educación regular incorporan cada vez más estudiantes de diversas características y necesidades educativas derivadas de múltiples condiciones. Asimismo, en las escuelas sobre todo de dependencia municipal, se ha observado el aumento de la diversidad sociocultural de estudiantes matriculados, producto de la inmigración de los últimos años hacia este país (Espinoza \& Valdebenito, 2018).

Las escuelas, por tanto, se han visto en la necesidad de generar estrategias para brindar respuestas educativas pertinentes para el alumnado diverso, entendiendo que todos son capaces de aprender en aulas regulares (Jorgensen, McSheehan \& Sonnenmeier, 2007). Esto, amparado por la entrada en vigencia de la Ley №20.845 de Inclusión Escolar, y del Decreto Oficial $N^{\circ} 83$ que aprueba criterios y orientaciones para efectuar adecuaciones en Educación Parvularia y Educación General Básica (Ministerio de Educación de Chile [MINEDUC], 2015).

En el contexto de aula, y como una necesidad identificada tanto a nivel mundial como a nivel nacional en la literatura, se espera que los profesores de educación regular ejecuten una enseñanza diferenciada para los estudiantes, considerando la diversidad de necesidades educativas presentes (Coady, Harper \& De Jong, 2016). Esta enseñanza diferenciada implica la ejecución de prácticas pedagógicas inclusivas, las que son parte de un abanico que va desde efectuar ajustes a la enseñanza general hasta la intervención específica en el aula de recursos (Bryant, Bryant \& Smith, 2019). Algunos autores señalan, que para que estas prácticas tengan éxito deben ser de alta calidad, diferenciadas según características del alumnado y con un monitoreo constante de la trayectoria del aprendizaje (Salend \& Duhaney, 2011). Por medio de la ejecución de dichas prácticas, se busca erradicar la segregación de estudiantes en las aulas de educación regular, considerando sus habilidades, características individuales y sociales (Engelbrecht, Savolainen, Nel, Koskela \& Okkolin, 2017). Por tanto, estas prácticas garantizan la participación y el acceso equitativo a una educación de calidad por parte de todo el alumnado presente en las escuelas (Kozleski, Artiles \& Waitoller, 2014).

No obstante, existe escasa claridad sobre las prácticas inclusivas que los docentes ejecutan para dar respuesta educativa a la diversidad de estudiantes y sus fundamentos; e incluso, algunos autores señalan que éstas podrían estar basadas en la improvisación (Jiménez, Aguilera, Valdés \& Hernández, 2017). Tampoco se evidencia claridad sobre los posibles matices en estas prácticas, considerando factores como el sexo, la formación pedagógica de base o los años de experiencia docente. A partir de esta situación, es que se plantea el siguiente estudio, el que tiene por objetivo identificar y comparar las prácticas pedagógicas inclusivas que los docentes de la Región de Coquimbo, Chile, declaran ejecutar en sus aulas.

Para dar a conocer esta investigación, en primer lugar, el artículo presenta las bases teóricas que sostienen este estudio, considerando evidencia publicada en idioma anglosajón e hispano. Posteriormente, se presenta la metodología del estudio, considerando: diseño, participantes, instrumentos para la recogida de información, procedimientos y procesamiento de los datos. Luego, se dan a conocer los resultados considerando los tipos de prácticas inclusivas, comparadas según agrupación por sexo, establecimiento educacional, formación de base de los docentes y años de experiencia. Por último, se dan 
a conocer la discusión y conclusiones, haciendo referencia a los principales hallazgos del estudio y su relevancia para el abordaje didáctico en las aulas con estudiantado diverso.

\section{MARCO TEÓRICO}

La investigación sobre prácticas pedagógicas inclusivas se ha llevado a cabo en distintas naciones, donde suelen presentarse algunas diferencias tanto a nivel de país como diferencias entre las escuelas donde se desempeñan los docentes participantes de estos estudios. Esta panorámica, por tanto, permite determinar algunos matices entre las prácticas pedagógicas, tanto desde lo que los docentes declaran como desde lo que efectivamente ejecutan dentro del aula con estudiantes de diversas características individuales, culturales, ritmos de aprendizaje e incluso necesidades educativas.

A continuación, se presentan en orden cronológico algunos estudios relevantes y recientes sobre la temática que aborda esta investigación, presentes en la literatura anglosajona. Posteriormente, se dan a conocer investigaciones en esta temática que se han llevado a cabo en países iberoamericanos, incluyendo Chile.

\subsection{ESTUDIOS ANGLOSAJONES SOBRE PRÁCTICAS PEDAGÓGICAS INCLUSIVAS}

En los últimos años se han generado estudios sobre prácticas pedagógicas inclusivas, que evidencian la diversidad de éstas incluso en un mismo contexto sociocultural. En Estados Unidos se ha observado que existe una gran diversidad de prácticas pedagógicas en las aulas inclusivas de escuelas de educación regular (Morningstar, Shogren, Lee \& Born, 2015). En este estudio, se identificaron metodologías diversas que van desde las tradicionales a las más innovadoras, diversidad en la forma de agrupación del estudiantado para efectuar las actividades de aprendizaje, prácticas centradas en la enseñanza directa desde el profesor, así como otras centradas en el estudiante, uso de diversidad de recursos de aprendizaje, así como también uso limitado de los mismos para la enseñanza. Este estudio, como otros existentes en la literatura, fue abordado desde un enfoque centrado en las necesidades educativas, más que centrado en la diversidad de estudiantado en el aula (Ryndak, Jackson \& White, 2013; Shogren, McCart, Lyon \& Sailor, 2015).

Respecto de contextos educativos como el canadiense, el estudio de Specht et al. (2015) entrega evidencias al respecto. La investigación consideró a una diversidad de 1.490 docentes en formación de Canadá. Los autores plantean que, en general, las mujeres declaran tener mayor eficacia para atender en aulas inclusivas que los hombres. Asimismo, aquellos docentes que han tenido prácticas y experiencia con estudiantado con necesidades educativas declaran hacer más prácticas inclusivas que aquellos que no. Por último, se halló que aquellos profesores en formación elemental o básica también suelen manifestar una mayor eficacia para practicar la inclusión en aula que los docentes en formación secundaria, quienes suelen compartir curricularmente con otros docentes de diferentes áreas.

Según algunos autores, las acciones pedagógicas para llevar a cabo la inclusión en el aula dependen en gran medida de aspectos culturales, y cómo desde el contexto se comprenden tanto la inclusión como la educación (Engelbrecht et al., 2017). En el estudio mencionado, los investigadores plantean que existen diferencias en países como Finlandia y Sudáfrica. Por una parte, en Finlandia la inclusión en la educación se concibe como un 
proceso sistemático y con un abordaje colaborativo. Por tanto, las prácticas pedagógicas en aula suelen considerar metodologías innovadoras empleadas de manera permanente, organización de pequeños grupos para fomentar el aprendizaje por pares, diferenciación de estrategias de enseñanza matizando según la complejidad de las habilidades, y el trabajo colaborativo entre pares docentes es habitual día a día. Por otro lado, en Sudáfrica, donde la educación y la inclusión suelen ser abordadas desde un enfoque más tradicional, las prácticas suelen considerar metodologías de enseñanza convencionales para todo el grupo de clase, y aunque suelen diversificar estrategias suelen ser muy específicas para el estudiantado con dificultades de aprendizaje, y con el apoyo de profesionales expertos en la materia. Esto deja entrever que desde el contexto sudafricano se observan las necesidades educativas aún desde un enfoque centrado en el déficit (Florian \& Black-Hawkins, 2011), mientras que en Finlandia el enfoque se centra en la diversidad en aula.

En contextos como Japón, (Yada \& Savolainen, 2017), los investigadores han hallado que los docentes japoneses no cuentan con las herramientas pedagógicas suficientes para enfrentar un aula inclusiva, debido a su escasa formación al respecto. Por lo mismo, el profesorado declara efectuar pocas prácticas de este tipo, lo cual va de la mano con presencia de ansiedad al enfrentarse a un aula que incluye a estudiantes con discapacidad o necesidades educativas diversas.

Por último y recientemente, en Estados Unidos, Hawkins, Crim, Ganz y Kennedy (2019) plantean que las aulas inclusivas requieren docentes preparados para atender a la diversidad de necesidades, como para intencionar el desarrollo de habilidades para el siglo XXI en todo el estudiantado. Esto consideraría la enseñanza diferenciada, la evaluación formativa para mejorar los procesos e implementar la tutoría por pares para potenciar un aprendizaje activo por parte del estudiantado.

\subsection{ESTUDIOS SOBRE PRÁCTICAS PEDAGÓGICAS INCLUSIVAS EN PAÍSES HISPANOS}

Un primer estudio a citar es el realizado por los autores Figueroa y Muñoz (2014), en Santiago de Chile. En este estudio, los investigadores concluyeron que las principales falencias en los establecimientos sería la escasa gestión del tiempo para generar ciertas instancias y espacios para dialogar y construir colectivamente respuestas innovadoras frente al sistema. Del mismo modo, los autores mencionan que una de las dificultades de los docentes inmersos en estos establecimientos es la falta de autocrítica hacia sus prácticas pedagógicas, puesto que en escasas ocasiones son capaces de mirar sus prácticas y culturas desde un punto de vista investigativo que aspire a mejorar y transformar sus dinámicas. Por esto, los autores plantean que es necesario favorecer la cultura y prácticas inclusivas bajo un enfoque sistemático de autoevaluaciones, de metacognición y concientización compartida en cada comunidad educativa.

Por otro lado, los autores Darretxe, Goikoetxea y Fernández (2013) analizaron las prácticas inclusivas y exclusivas en dos centros educativos de educación primaria y educación secundaria del País Vasco, España. Dentro de los principales hallazgos, se encontró que ambos establecimientos comparten una cultura y una práctica educativa bastante alejada a una inclusión efectiva, según los autores. Además, estos mencionan que al no conocer en profundidad una cultura inclusiva es probable que los docentes desconozcan estrategias para enfrentar una práctica educativa inclusiva dentro del aula. En conclusión, manifiestan que para poder mejorar las prácticas inclusivas el profesorado debe procurar un cambio 
cultural y organizativo que nazca de las mismas instituciones, por lo que es relevante que toda la comunidad concientice una cultura escolar inclusiva para que posteriormente surjan prácticas inclusivas dentro del aula.

Serrato y García (2014) también efectuaron un estudio sobre prácticas inclusivas en México, enmarcado en la evaluación de un programa de intervención que fue ejecutado para promover dichas prácticas. Los resultados arrojaron que luego de la aplicación del instrumento antes y después de la intervención, los docentes han realizado cambios importantes en sus prácticas, sobre todo, en la sensibilización ante la diversidad y la reflexión acerca de su quehacer pedagógico. Asimismo, puede verse que existen menos discrepancias en las puntuaciones de los promedios entre la prueba de auto-reporte y la de observación en el post test que en el pre test. Esto quiere decir que hay mayor concordancia entre lo que los docentes reportan hacer y lo que se observa en la práctica después del proceso de intervención. Esto puede indicar, según los autores, que el docente en un principio puede haber sobrevalorado sus prácticas inclusivas y que, debido al curso y la intervención, hicieron una evaluación posterior más objetiva de las mismas.

En 2015, García, Romero, Rubio, Jocabed y Martínez dieron a conocer su estudio, donde se compararon los tipos de prácticas de docentes en servicio de educación especial y regular, también en México. Dentro de los principales hallazgos se encontró que, en general, los docentes de educación especial y regular declararon tener prácticas altamente favorecedoras a la inclusión. No obstante, los investigadores señalan que los resultados deben ser considerados con cierta reserva, puesto que los docentes tienden a sobrecalificar su trabajo y esto podría provocar ciertos sesgos en la investigación. Las conclusiones de los investigadores señalan que, si bien los docentes de las escuelas especiales eran más individualistas en su labor, estos realizaban mayores y mejores prácticas inclusivas que los docentes de colegios regulares. Esta tendencia se da posiblemente por las condiciones de trabajo, puesto que los profesores de educación especial suelen contar con grupos pequeños de estudiantes, tienen experiencia en el trabajo con estudiantes con discapacidad, experiencia en el diseño y puesta en práctica de las adecuaciones curriculares y la aceptación de estudiantes con discapacidad.

Posteriormente, en España, los autores Gónzalez, Martín, Poy y Jeraro (2016) efectuaron un estudio donde participaron 402 profesores españoles. Dentro de los principales hallazgos, se visualiza que los docentes emplean escasas prácticas pedagógicas inclusivas y que se muestran resistentes a modificar su quehacer pedagógico dentro del aula. De la misma forma, los autores señalan que el $88 \%$ de los docentes casi nunca realizan adecuaciones curriculares o si las realizan estas son poco significativas. Respecto a los resultados considerando el género, no se hallaron diferencias significativas respecto de la favorabilidad para implementar prácticas inclusivas. Con relación a los años de experiencia docente, son los profesores con menos experiencia los que declaran mayor favorabilidad hacia estas prácticas, aunque este resultado no fue significativo.

Finalmente, es interesante citar el estudio realizado por los autores Mellado, Chaucono, Hueche y Aravena (2017), que se llevó a cabo en un colegio de educación regular con programa de integración escolar de la Región de Los Ríos, Chile. Se hallaron diferencias entre las respuestas de los docentes de aula regular y los docentes de educación especial, ya que en general los docentes de aula regular declaraban de manera implícita segregar a los niños con necesidades educativas especiales (NEE). Dado esto, los mismos docentes de aula regular declaraban bajas expectativas en referencia a este grupo de estudiantes, lo 
cual, según los autores explica la falta de planificaciones diversificadas y metodologías inclusivas que involucren el aprendizaje de todo el estudiantado. Respecto de los docentes de educación especial, los autores explican que manifiestan más prácticas inclusivas debido a que poseen mayor conocimiento del concepto de inclusión y lo que implica, gracias a su formación de base.

\subsection{SOBRE ESTE ESTUDIO}

Tomando en cuenta los estudios revisados previamente, se revela la necesidad de abordar como objeto de estudio las prácticas inclusivas de los profesores en las aulas chilenas, profundizando y ampliando el conocimiento desde los hallazgos que variados estudios previos han revelado. El propósito de este estudio, particularmente, es indagar sobre las prácticas pedagógicas inclusivas que declaran efectuar los docentes, estableciendo comparaciones entre los profesores participantes según los siguientes criterios de agrupación: colegio en el que se desempeña, sexo, formación pedagógica de base y años de experiencia. Estas prácticas pedagógicas se abordan diferenciadamente, considerando la planificación, las metodologías, la evaluación, el uso pedagógico del tiempo y los recursos para el aprendizaje.

\section{METODOLOGÍA DEL ESTUDIO}

Este estudio se llevó a cabo desde un enfoque metodológico cuantitativo, por comparación de grupos. Los participantes del estudio corresponden a 88 profesores y profesoras ( 21 hombres y 67 mujeres) pertenecientes a dos colegios de las comunas de La Serena y Coquimbo, en la Región de Coquimbo, Chile. Dichos colegios se ubican en zonas urbanas de desarrollo sociocultural medio-bajo, con altos niveles de población inmigrante y con un desarrollo económico descendido. Los colegios imparten clases desde educación preescolar hasta educación media, siendo uno de los colegios de educación media de tipo humanista, y el otro de educación media técnico profesional. Ambos colegios cuentan con estudiantes inmigrantes, y además con Programa de Integración Escolar (PIE) que atiende a estudiantes con NEE asociadas a diferentes diagnósticos clínicos.

Se llevó a cabo un muestreo no probabilístico intencionado, en el que, según los objetivos del estudio, se escoge deliberadamente a los participantes de este. Como criterios de inclusión se tomó en cuenta que los profesores ejercieran clases en aula. Los participantes tenían una formación de base como docentes de Educación Parvularia $(n=7$, $8 \%)$, Educación General Básica $(n=19,21,6 \%)$, Educación Media en las diversas áreas $(n=36,40,9 \%)$, docentes de Educación Diferencial $(n=23,26,1 \%)$, y aquellos profesores que laboraban en educación técnico profesional, aunque no tenían una formación pedagógica de base $(n=3,3,4 \%)$. Tenían entre 0 y 40 años de experiencia en docencia. Se excluyeron a todos aquellos docentes de estos colegios que no ejercieran labores pedagógicas directas con los estudiantes en aula.

A continuación, se presenta la Tabla 1, donde se caracteriza a los profesores participantes de este estudio considerando sus rangos de edad. 
Tabla 1. Profesores participantes en el estudio según rangos de edad

\begin{tabular}{|c|c|c|c|c|}
\hline Rango de edad & $\boldsymbol{n} \mathbf{C 1}$ & $\boldsymbol{n} \mathbf{C 2}$ & $\boldsymbol{n}$ total & $\boldsymbol{\%}$ total \\
\hline 20-30 años & 10 & 7 & 17 & 19.3 \\
\hline 31-40 años & 27 & 19 & 46 & 52.3 \\
\hline 41-50 años & 11 & 6 & 17 & 19.3 \\
\hline + de 50 años & 6 & 2 & 8 & 9.1 \\
\hline Total & 54 & 34 & 88 & 100 \\
\hline
\end{tabular}

Nota: $n=$ Cantidad de participantes. $\mathrm{C} 1=$ Colegio de la ciudad de La Serena. $\mathrm{C} 2=$ Colegio de la ciudad de Coquimbo.

Fuente. Elaboración propia.

\subsection{INSTRUMENTOS}

Primeramente, se empleó una ficha de antecedentes sociodemográficos para consignar información de los participantes sobre: el colegio en que se desempeña, sexo, edad, formación pedagógica de base, años de experiencia, entre otros antecedentes. Esta información permitió la posterior organización de los grupos para los análisis de datos, considerando estos criterios.

Para obtener información sobre las prácticas pedagógicas inclusivas que los docentes declaran emplear, se aplicó el instrumento Guía de Evaluación de Prácticas Inclusivas en el Aula (GEPIA) de los autores García, Romero y Escalante (2011). Originalmente, este instrumento considera las modalidades de auto-reporte y observación. Ambas versiones reúnen elementos ajustados del instrumento Índice de Inclusión de Booth y Ainscow (2000, 2011). El propósito del instrumento es recoger información respecto de las prácticas inclusivas ejecutadas por la planta docente en relación con dos dimensiones: prácticas inclusivas pedagógicas en aula, y cultura y políticas inclusivas. Cabe mencionar, que en este estudio se empleó la modalidad de auto-reporte, debido a que posee mayor nivel de confiabilidad (.88) que el instrumento de observación (.80) (García et al., 2015). Asimismo, por razones de tiempo de acceso a los establecimientos educacionales, el que fue limitado. El formato de esta versión corresponde a una escala de frecuencia que posee una progresión ascendente que va desde el nivel 0 "nunca" al nivel 3 "siempre".

Para los fines de este estudio, se consideraron los datos recogidos en la dimensión del instrumento: prácticas inclusivas de tipo pedagógica que los profesores declaran emplear dentro del aula. Esta dimensión de prácticas inclusivas se compone de cinco ámbitos, a saber: Condiciones físicas del aula (uso y aprovechamiento de los recursos materiales para promover una mejor enseñanza dentro del aula), Planeación (organización previa por parte de los docentes respecto a las evaluaciones y las adecuaciones curriculares), Uso del tiempo (utilización y gestión del tiempo en relación a los ritmos de aprendizaje de cada estudiante), Metodología (forma en la que el docente presenta y organiza los contenidos durante la clase) y Evaluación (forma de evaluar respetando el ritmo de cada estudiante). 


\subsection{PROCEDIMIENTOS PARA LA APLICACIÓN}

Los instrumentos fueron aplicados de forma presencial, en un único encuentro en cada establecimiento educacional, de una duración aproximada de 30 minutos. El trabajo de campo estuvo a cargo de dos investigadoras previamente instruidas para el correcto empleo de los instrumentos. Además, se informó previamente al profesorado sobre el estudio, quienes dieron su consentimiento escrito y previamente informado para participar en él.

\subsection{PROCESAMIENTO DE LOS DATOS OBTENIDOS}

La información recogida por medio de los instrumentos fue vaciada en una base de datos, empleando el software Statistical Package for the Social Sciences SPSS, versión 20.0. Para los análisis se utilizaron las puntuaciones brutas o directas obtenidas. Desde ellas se calcularon los promedios por cada ámbito de práctica pedagógica inclusiva, con el fin de que la cantidad de participantes por grupo no afectara los análisis a efectuar.

Primeramente, se llevaron a cabo análisis exploratorios para verificar la normalidad en la distribución de datos. Posteriormente, se efectuaron pruebas no paramétricas para comparar grupos: Prueba de U de Mann-Whitney para comparar dos muestras independientes, y Prueba de Kruskall-Wallis para comparar tres o más muestras independientes. Esto se fundamenta, pues estas pruebas no paramétricas son empeladas cuando la distribución de datos no es normal (López \& Diez, 2017). Esta situación suele suceder, cuando se recoge información por medio de instrumentos del tipo escala, generando en la base de datos que las variables sean de tipo ordinal (Camacho, 2008). El nivel de confianza para los análisis se adoptó en $95 \%$ (error alfa .05).

\section{RESULTADOS}

La presentación de los resultados se efectúa considerando los ámbitos de las prácticas inclusivas determinadas por el instrumento empleado, a considerar: Condiciones físicas del aula, planeación, uso del tiempo, metodología y evaluación. Cada ámbito fue analizado según las distintas agrupaciones de los participantes, a saber: agrupación por colegio en el que trabaja el docente, agrupación por sexo, por formación pedagógica de base y según los años de experiencia docente.

\subsection{PRÁCTICAS PEDAGÓGICAS INCLUSIVAS DECLARADAS SEGÚN COLEGIO AL QUE PERTENECEN LOS DOCENTES}

La Tabla 2 presenta información sobre las diferencias existentes entre grupos, considerando si los profesores participantes del estudio pertenecían al colegio de Coquimbo o al colegio de La Serena. Según los resultados, existe una diferencia estadísticamente significativa entre colegios según las prácticas pedagógicas inclusivas asociadas a la evaluación ( $p \leq$ .05). En esta diferencia entre grupos, se evidenció que el colegio de Coquimbo declara efectuar más ajustes en el ámbito de evaluación del aprendizaje, por sobre el colegio de La Serena. 
A pesar de que en el resto de los ámbitos el colegio de Coquimbo declara efectuar más prácticas pedagógicas inclusivas, no se evidencian diferencias estadísticamente significativas entre ambos establecimientos educacionales.

Tabla 2. Diferencias entre prácticas pedagógicas declaradas por los profesores agrupados según colegio

\begin{tabular}{|c|c|c|c|c|c|c|c|}
\hline \multirow[b]{3}{*}{ Ámbitos } & \multicolumn{4}{|c|}{ Participantes } & \multirow[b]{3}{*}{$U$} & \multirow[b]{3}{*}{ Z } & \multirow[b]{3}{*}{$p$} \\
\hline & \multicolumn{2}{|c|}{$\begin{array}{c}\text { Colegio de Coquimbo } \\
(n=34)\end{array}$} & \multicolumn{2}{|c|}{$\begin{array}{l}\text { Colegio de La Serena } \\
\qquad(n=54)\end{array}$} & & & \\
\hline & $\begin{array}{c}\text { Rango } \\
\text { promedio }\end{array}$ & $\begin{array}{l}\text { Suma de } \\
\text { rangos }\end{array}$ & $\begin{array}{c}\text { Rango } \\
\text { promedio }\end{array}$ & $\begin{array}{l}\text { Suma de } \\
\text { rangos }\end{array}$ & & & \\
\hline $\begin{array}{c}\text { Prácticas } \\
\text { pedagógicas } \\
\text { inclusivas (total) }\end{array}$ & 49.50 & 1683 & 41.35 & 2233 & 748 & -1.459 & .145 \\
\hline $\begin{array}{c}\text { Ámbito } 1 \\
\text { Condiciones físicas } \\
\text { del aula }\end{array}$ & 50.71 & 1724 & 40.59 & 2192 & 707 & -1.837 & .066 \\
\hline $\begin{array}{c}\text { Ámbito } 2 \\
\text { Planificación }\end{array}$ & 47.41 & 1612 & 42.67 & 2304 & 819 & -.856 & .392 \\
\hline $\begin{array}{c}\text { Ámbito } 3 \\
\text { Uso del tiempo }\end{array}$ & 48.60 & 1652.5 & 41.92 & 2263.5 & 778.5 & -1.242 & .214 \\
\hline $\begin{array}{c}\text { Ámbito } 4 \\
\text { Metodología }\end{array}$ & 44.91 & 1527 & 44.24 & 2389 & 904 & -.121 & .904 \\
\hline $\begin{array}{l}\text { Ámbito } 5 \\
\text { Evaluación }\end{array}$ & 52.41 & 1782 & 39.52 & 2134 & 649 & -2.360 & $.018^{*}$ \\
\hline
\end{tabular}

Nota: $n=$ número de participantes del grupo, $U=\mathrm{U}$ de Mann-Whitney, $Z=$ Valor tipificado, $p=$ Significación. $* p \leq .05, * * p \leq .01, * * * p \leq .001$

Fuente. Elaboración propia.

\subsection{PRÁCTICAS PEDAGÓGICAS INCLUSIVAS DECLARADAS SEGÚN SEXO}

En la Tabla 3 se consignan los resultados sobre las diferencias existentes entre grupos según sexo. Los análisis revelan que existe una diferencia estadísticamente significativa entre hombres y mujeres, considerando la totalidad de las prácticas pedagógicas inclusivas consultadas $(p \leq .01)$. Al efectuar un análisis más específico, esta diferencia significativa entre grupos en la totalidad de prácticas pedagógicas inclusivas se debe mayormente a las diferencias entre grupos que se generan en el ámbito de metodología $(p \leq .05)$ y evaluación $(p \leq .05)$. En el ámbito de planificación no se evidenció diferencia significativa entre grupos, aunque se observó una significación marginal $(p=.051)$. La diferencia significativa entre grupos según sexo, evidencia que las mujeres declaran efectuar más ajustes a nivel metodológico y de evaluación del aprendizaje en aula que los hombres.

A pesar de que las mujeres declaran además efectuar más prácticas pedagógicas inclusivas respecto de las condiciones físicas del aula y uso del tiempo, no se evidencian diferencias estadísticamente significativas entre ambos grupos según sexo en dichos ámbitos. 
Tabla 3. Diferencias entre prácticas pedagógicas declaradas por los profesores agrupados según sexo

\begin{tabular}{|c|c|c|c|c|c|c|c|}
\hline \multirow[b]{3}{*}{ Ámbitos } & \multicolumn{4}{|c|}{ Participantes } & \multirow[b]{3}{*}{$U$} & \multirow[b]{3}{*}{$\mathrm{Z}$} & \multirow[b]{3}{*}{$p$} \\
\hline & \multicolumn{2}{|c|}{$\begin{array}{c}\text { Mujeres } \\
(n=67) \\
\end{array}$} & \multicolumn{2}{|c|}{$\begin{array}{c}\text { Hombres } \\
(n=21)\end{array}$} & & & \\
\hline & $\begin{array}{l}\text { Rango } \\
\text { promedio }\end{array}$ & $\begin{array}{l}\text { Suma de } \\
\text { rangos }\end{array}$ & $\begin{array}{l}\text { Rango } \\
\text { promedio }\end{array}$ & $\begin{array}{l}\text { Suma de } \\
\text { rangos }\end{array}$ & & & \\
\hline $\begin{array}{c}\text { Prácticas } \\
\text { pedagógicas } \\
\text { inclusivas (total) }\end{array}$ & 48.48 & 3248 & 31.81 & 668 & 437 & -2.612 & $.009 * *$ \\
\hline $\begin{array}{c}\text { Ámbito } 1 \\
\text { Condiciones } \\
\text { físicas del aula }\end{array}$ & 47.15 & 3159 & 36.05 & 757 & 526 & -1.766 & .077 \\
\hline $\begin{array}{c}\text { Ámbito } 2 \\
\text { Planificación }\end{array}$ & 47.45 & 3179 & 35.10 & 737 & 506 & -1.950 & .051 \\
\hline $\begin{array}{c}\text { Ámbito } 3 \\
\text { Uso del tiempo }\end{array}$ & 46.63 & 3124 & 37.71 & 792 & 561 & -1.449 & .147 \\
\hline $\begin{array}{c}\text { Ámbito } 4 \\
\text { Metodología }\end{array}$ & 48.32 & 3237.5 & 32.31 & 678.5 & 447.5 & -2.530 & $.011^{*}$ \\
\hline $\begin{array}{l}\text { Ámbito } 5 \\
\text { Evaluación }\end{array}$ & 48.28 & 3235 & 32.43 & 681 & 450 & -2.541 & $.011 *$ \\
\hline
\end{tabular}

Nota: $n=$ número de participantes del grupo, $U=\mathrm{U}$ de Mann-Whitney, $Z=$ Valor tipificado, $p=$ Significación. $* p \leq .05, * * p \leq .01, * * * p \leq .001$.

Fuente. Elaboración propia.

\subsection{PRÁCTICAS PEDAGÓGICAS INCLUSIVAS SEGÚN LA FORMACIÓN DE BASE DE LOS DOCENTES}

La Tabla 4 presenta información sobre diferencias entre grupos, considerando su formación de base como docente. Según los resultados, existe una diferencia estadísticamente significativa entre los grupos en la totalidad de prácticas pedagógicas inclusivas $(p \leq .05)$. Al efectuar un análisis más específico, el ámbito que genera esta diferencia significativa entre grupos es la metodología $(p \leq .01)$.

En el ámbito de metodología, los grupos que presentan diferencias significativas entre sí, son: profesorado de Educación Diferencial y docentes sin formación pedagógica de base $(p=.021)$, profesorado de Educación Diferencial y profesorado de Educación Media $(p=.023)$, profesorado de Educación Diferencial y profesorado de Educación Parvularia $(p=.025)$, profesorado de Educación General Básica y docentes sin formación pedagógica de base $(p=.026)$, profesorado de Educación General Básica y profesorado de Educación Parvularia $(p=.036)$, profesorado de Educación General Básica y profesorado de Educación Media ( $p=.044)$, (ver Figura 1).

No se evidencian diferencias estadísticamente significativas entre grupos según formación pedagógica de base en el resto de prácticas pedagógicas inclusivas. 
Tabla 4. Diferencias entre prácticas pedagógicas declaradas por los profesores según formación de base

\begin{tabular}{|c|c|c|c|c|c|c|c|}
\hline \multirow[b]{2}{*}{ Ámbitos } & \multicolumn{5}{|c|}{ Rango promedio del profesorado } & \multirow[b]{2}{*}{$\chi^{2}$} & \multirow[b]{2}{*}{$p$} \\
\hline & $\begin{array}{c}\text { Ed. } \\
\text { Preescolar } \\
(n=7)\end{array}$ & $\begin{array}{c}\text { Ed. Gral. } \\
\text { Básica } \\
(n=19)\end{array}$ & $\begin{array}{c}\text { Ed. } \\
\text { Media } \\
(n=36)\end{array}$ & $\begin{array}{c}\text { Ed. } \\
\text { Diferencial } \\
(n=23)\end{array}$ & $\begin{array}{c}\text { Sin formación } \\
\text { pedagógica } \\
(n=3)\end{array}$ & & \\
\hline $\begin{array}{c}\text { Prácticas } \\
\text { pedagógicas } \\
\text { inclusivas (total) }\end{array}$ & 33.86 & 54,47 & 38.47 & 52.02 & 20.83 & 10.713 & $.030^{*}$ \\
\hline $\begin{array}{c}\text { Ámbito } 1 \\
\text { Condiciones } \\
\text { físicas del aula }\end{array}$ & 43.43 & 55.32 & 38.51 & 47.70 & 25.83 & 7.595 & .108 \\
\hline $\begin{array}{c}\text { Ámbito } 2 \\
\text { Planificación }\end{array}$ & 36.71 & 53.37 & 37.89 & 51.83 & 29.67 & 8.395 & .078 \\
\hline $\begin{array}{c}\text { Ámbito } 3 \\
\text { Uso del tiempo }\end{array}$ & 29.07 & 50.50 & 42.72 & 49.65 & 24.33 & 7.105 & .130 \\
\hline $\begin{array}{c}\text { Ámbito } 4 \\
\text { Metodología }\end{array}$ & 29.61 & 53.24 & 38.76 & 54.17 & 18.33 & 13.075 & $.011 * *$ \\
\hline $\begin{array}{l}\text { Ámbito } 5 \\
\text { Evaluación }\end{array}$ & 44.43 & 52.26 & 40.21 & 46.26 & 33.50 & 3.602 & .462 \\
\hline
\end{tabular}

Nota: $n=$ Número de participantes del grupo, $g l=4$ grados de libertad, $\chi^{2}=$ Chi cuadrado, $p=$ Significación. $* p \leq .05, * * x \leq .01, * * * p \leq .001$.

Fuente: Elaboración propia.

Figura 1. Gráfico boxplt de comparación entre grupos de profesores en el ámbito de Metodología. según su formación de base.

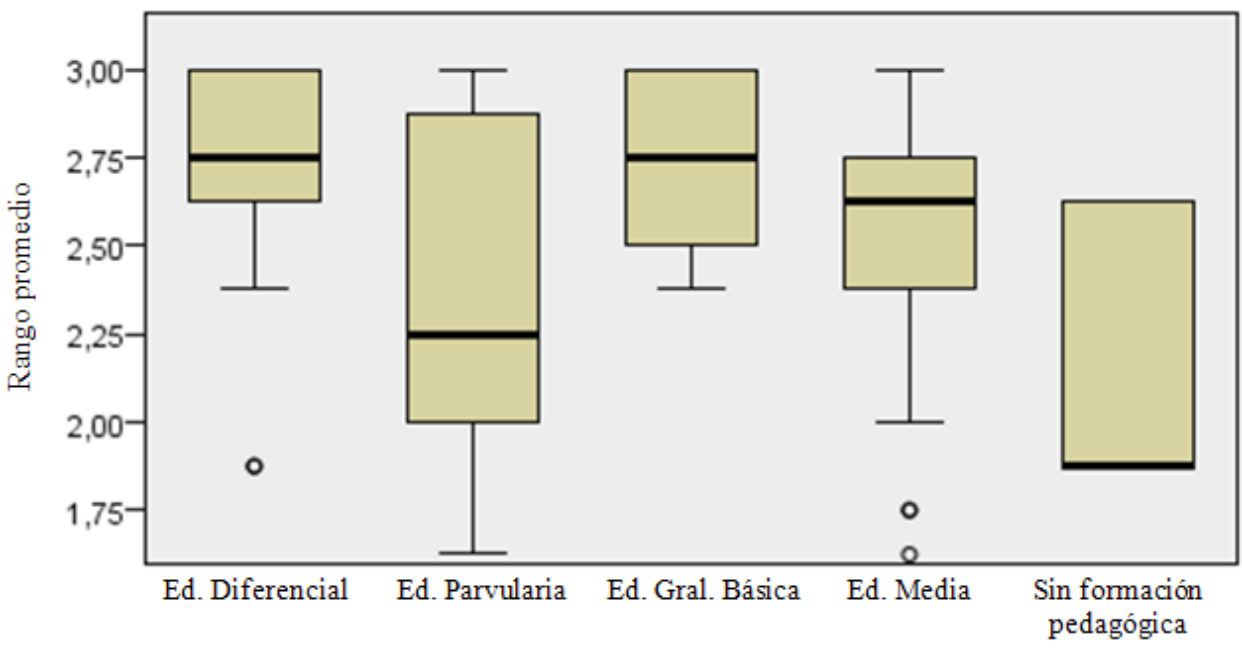

Fuente: Elaboración propia. 


\subsection{PRÁCTICAS PEDAGÓGICAS INCLUSIVAS SEGÚN LOS AÑOS DE EXPERIENCIA DE LOS DOCENTES}

En la Tabla 5 se consignan los resultados de los análisis sobre diferencias entre grupos, considerando los años de experiencia de los docentes en esta labor. De acuerdo a la información obtenida, existe una diferencia estadísticamente significativa entre los grupos en la totalidad de prácticas pedagógicas inclusivas $(p \leq .05)$. Al efectuar un análisis más detallado, el ámbito que genera esta diferencia significativa entre grupos es condiciones físicas del aula $(p \leq .05)$.

En el ámbito de condiciones físicas del aula, los grupos de profesorado que presentan diferencias significativas entre sí, son: profesorado en rango de 0 a 2 años de experiencia y profesorado en rango de 21 a 30 años de experiencia $(p=.006)$, profesorado en rango de 11 a 20 años de experiencia y profesorado en rango de 21 a 30 años de experiencia $(p=.014)$, profesorado en rango de 0 a 2 años de experiencia y profesorado en rango de 31 a 40 años de experiencia $(p=.022)$, (ver Figura 2).

No se evidencian diferencias estadísticamente significativas entre grupos según los años de experiencia docente en el resto de prácticas pedagógicas inclusivas.

Tabla 5. Diferencias entre prácticas pedagógicas declaradas por los profesores según años de experiencia docente

\begin{tabular}{|c|c|c|c|c|c|c|c|c|}
\hline \multirow[b]{2}{*}{ Ámbitos } & \multicolumn{6}{|c|}{ Rango promedio del profesorado } & \multirow[b]{2}{*}{$\chi^{2}$} & \multirow[b]{2}{*}{$p$} \\
\hline & $\begin{array}{c}0-2 \text { años } \\
(n=7)\end{array}$ & $\begin{array}{c}3-5 \text { años } \\
(n=20)\end{array}$ & $\begin{array}{c}6-10 \text { años } \\
(n=30)\end{array}$ & $\begin{array}{c}11-20 \text { años } \\
(n=21)\end{array}$ & $\begin{array}{c}21-30 \text { años } \\
(n=6)\end{array}$ & $\begin{array}{c}31-40 \text { años } \\
(n=4)\end{array}$ & & \\
\hline $\begin{array}{c}\text { Prácticas } \\
\text { pedagógicas } \\
\text { inclusivas (total) }\end{array}$ & 23.14 & 44.90 & 45.08 & 40.43 & 65.92 & 64.75 & 12.209 & $.032 *$ \\
\hline $\begin{array}{c}\text { Ámbito } 1 \\
\text { Condiciones } \\
\text { físicas del aula }\end{array}$ & 27.5 & 45.00 & 46.08 & 37.60 & 66.25 & 63.50 & 11.685 & $.039 *$ \\
\hline $\begin{array}{c}\text { Ámbito } 2 \\
\text { Planificación }\end{array}$ & 30.71 & 39.88 & 45.72 & 43.38 & 61.17 & 63.50 & 7.689 & .174 \\
\hline $\begin{array}{c}\text { Ámbito } 3 \\
\text { Uso del tiempo }\end{array}$ & 25.21 & 47.83 & 44.23 & 41.90 & 60.00 & 54.00 & 7.892 & .162 \\
\hline $\begin{array}{c}\text { Ámbito } 4 \\
\text { Metodología }\end{array}$ & 23.00 & 45.10 & 43.92 & 43.79 & 60.00 & 64.00 & 9.725 & .083 \\
\hline $\begin{array}{c}\text { Ámbito } 5 \\
\text { Evaluación }\end{array}$ & 30.50 & 46.68 & 44.60 & 39.93 & 58.67 & 60.13 & 6.565 & .225 \\
\hline
\end{tabular}

Nota: $n=$ Número de participantes del grupo, $g l=5$ grados de libertad, $\chi^{2}=$ Chi cuadrado, $p=$ Significación. $* p \leq .05, * * p \leq .01, * * * p \leq .001$.

Fuente. Elaboración propia. 
Figura 2. Gráfico boxplt de comparación entre grupos de profesores en el ámbito de Condiciones físicas del aula. según años de experiencia docente.

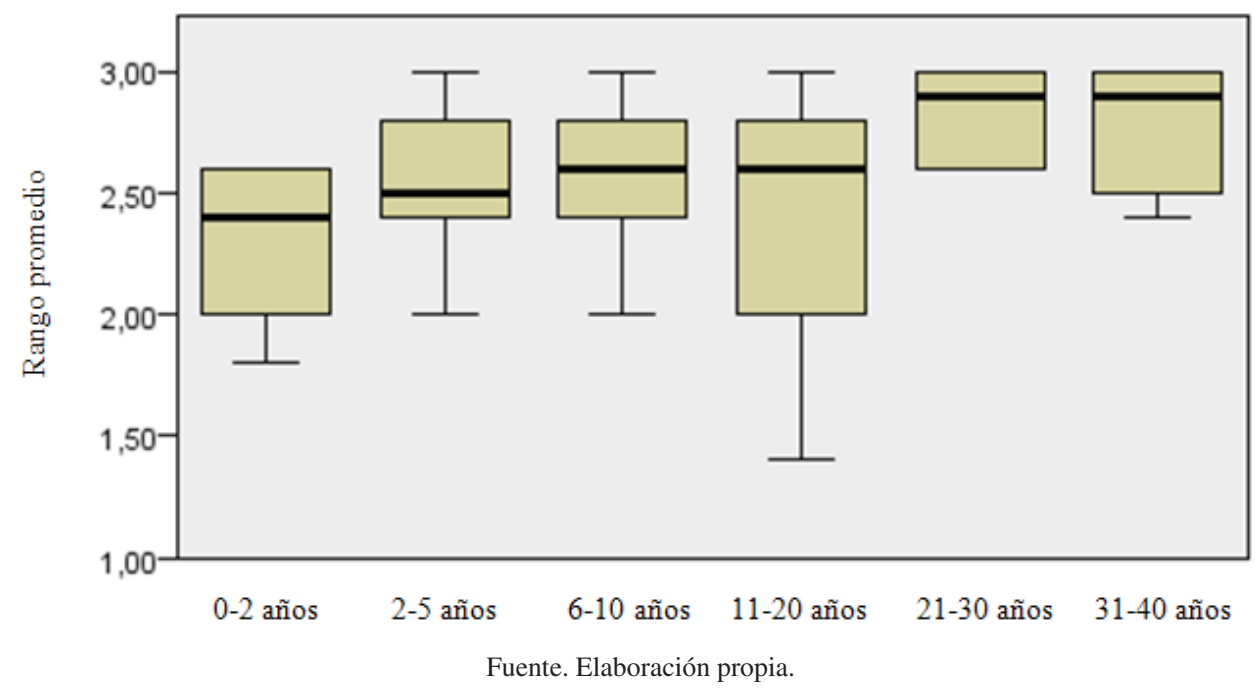

\section{DISCUSIÓN}

A partir de la literatura revisada y de los resultados obtenidos en este estudio, se evidencia que existen algunas diferencias significativas entre prácticas pedagógicas inclusivas que los docentes declaran efectuar, asociadas con la planificación, las metodologías de enseñanza y aprendizaje, la evaluación, el uso del tiempo, y de los recursos y espacios para el aprendizaje. Asimismo, las diferencias en estas prácticas en los participantes de este estudio presentan algunos matices, dependiendo de su sexo, del colegio en que se encuentren trabajando, así como de su formación pedagógica de base y años de experiencia docente.

Respecto de las diferencias entre prácticas pedagógicas inclusivas según establecimiento educacional, las halladas en este estudio son modestas, aunque en las prácticas de ajuste a la evaluación se halló una diferencia significativa entre docentes de ambos colegios. Esta modesta diferencia puede deberse a que ambos colegios son de similares características, y se encuentran ubicados en similares zonas de vulnerabilidad social en las ciudades, situación descrita en el apartado de Metodología. Por ende, los estudiantes con los que los docentes interactúan pedagógicamente son de características semejantes y los ajustes pedagógicos para dar la respuesta educativa a esa diversidad serían también similares entre sí. Además, al tener ambos colegios un funcionamiento y estructura parecidos, es probable que compartan un enfoque o paradigma común, desde el cual emanan prácticas pedagógicas semejantes. Como se evidenció en el estudio de Darretxe et al. (2013), dependiendo de si el enfoque docente es centrado en el déficit, en las necesidades educativas del estudiante o en la diversidad del aula, es que se derivarán cierto tipo de prácticas pedagógicas inclusivas. Por otro lado, estudios como el de Morningstar et al. (2015) señalan que hay ciertas diferencias entre establecimientos educacionales incluso en un mismo país, lo que manifestaría matices de las diversas prácticas pedagógicas inclusivas, conformando un 
abanico y pudiendo estas diferencias llegar a ser o no relevantes. Asimismo, más notorias serán las diferencias entre estas prácticas pedagógicas si los contextos en que se encuentran los establecimientos son cultural y geográficamente diferentes, como se evidencia en el estudio de Engelbrecht et al. (2017).

Con relación a las diferencias de prácticas pedagógicas inclusivas considerando si el docente es hombre o mujer, en esta investigación se hallaron diferencias significativas en las prácticas de metodología, de evaluación y marginalmente de planificación. En el uso del tiempo y de recursos o espacios para el aprendizaje no se encontraron diferencias significativas, aunque igualmente las mujeres declararon efectuar más ajustes en este tipo de prácticas que los hombres. Las diferencias de género no parecen tener que ver con la cantidad de participantes por grupo, puesto que en otros estudios también se evidencia una mayor cantidad de mujeres que de hombres en la muestra (González et al., 2016; Specht et al., 2016). Además, en los estudios se suele controlar metodológicamente esta inequidad empleando las medias de los puntajes por dimensión y participante para los análisis. En el estudio de Specht et al. (2016) y de Chiner (2011) se halló, al igual que en este estudio, que las mujeres tienden a efectuar más prácticas inclusivas. Esto podría deberse a que las prácticas pedagógicas de las mujeres suelen estar más centradas en el estudiante (Flores, 2007), lo que podría deberse a su formación considerando que la mayor parte del profesorado de educación preescolar y escolar son mujeres. Asimismo, a que manifiestan mayores actitudes hacia la inclusión de los estudiantes (Garzón, Calvo y Orgas, 2016; Vicuña, 2013). Por otro lado, en el estudio de González et al. (2016) no se hallaron diferencias en la valoración de estas prácticas según género; es decir, hombres y mujeres valoran como importante la ejecución de prácticas pedagógicas inclusivas en el aula. Sin embargo, esto no significaría que las ejecuten efectivamente, pues es allí donde aparecerían las diferencias por género.

Asociado a la formación docente de base de los participantes de este estudio, se halló que el profesorado con formación en Pedagogía en Educación Diferencial y Pedagogía en Educación General Básica declara efectuar más prácticas pedagógicas inclusivas que el profesorado con formación en Pedagogía en Educación Media, Pedagogía en Educación Parvularia y los docentes sin formación pedagógica. Este patrón de diferencias señalado se da en todas las prácticas pedagógicas consideradas en el estudio, pero aparece como significativa en la práctica pedagógica específica de metodología. Este hallazgo es congruente con otros estudios (Chiner, 2011; García et al., 2015; Mellado et al., 2017; Specht et al., 2016). Según estos estudios, esta diferencia se debería a que los profesores de Educación Diferencial y de Educación General Básica tienden a manifestar una mayor aceptación de los estudiantes, sobre todo con necesidades educativas y discapacidades, pues ya han trabajado en cierto grado con ellos. Asimismo, tienden a enfocar su trabajo en estudiantes con edades tempranas que requieren de por sí ajustes metodológicos para el aprendizaje debido a razones evolutivas. Por otro lado, los docentes de Pedagogía en Educación Parvularia, Pedagogía en Educación Media y sin formación pedagógica se desempeñan en niveles educativos que, en Chile, suelen tener una menor incorporación de estudiantes con necesidades educativas; y, por otra parte, es posible que no reconozcan aún como una razón de ajuste de la metodología de enseñanza aquellas necesidades educativas asociadas a la diversidad étnica, sociocultural y las características individuales del estudiantado.

Respecto de las prácticas pedagógicas inclusivas que el profesorado declara ejecutar según los años de experiencia docente, en este estudio se halló que los profesores más 
antiguos que tenían entre 21 a 40 años de experiencia, manifestaron efectuar más ajustes a la enseñanza. Estas diferencias se evidencian en todas las prácticas pedagógicas consideradas en el estudio, aunque es estadísticamente significativa solamente en la dimensión condiciones físicas del aula. Por tanto, en los participantes de este estudio se observa que, mientras más jóvenes son, tienden a declarar que ejecutan menos ajustes a los recursos y condiciones físicas para el aprendizaje, mientras que cuando van aumentando en edad y experiencia, tienden a declarar más ajustes de este tipo. Esto podría deberse a que la experiencia juega un papel importante (Chiner, 2011), puesto que al estar el docente viviendo constantemente situaciones de atención a la diversidad se promueven en él actitudes más favorables hacia esta, por lo que los niveles de ansiedad frente a lo desconocido disminuyen (Granada, Pomés \& Sanhueza, 2013). Asimismo, debido a que los docentes más mayores suelen manifestar que se encuentran más preparados para enfrentar a la diversidad y la discapacidad (Suriá, 2012). Por otro lado, aunque hay estudios que señalan que la edad no es un factor que determina la actitud hacia la inclusión de estudiantes (Garzón et al., 2016; Granada et al., 2013), la investigación de Vicuña (2013) plantea que los profesores mayores tienen más actitudes positivas hacia la inclusión, lo que podría impulsar que declaren efectuar más prácticas pedagógicas inclusivas. No obstante, es importante reiterar que la diferencia por edad se evidenció significativamente en la dimensión condiciones físicas del aula, lo que deja entrever que los ajustes que efectúan los docentes más mayores son a los elementos externos, los materiales y los ambientes, pero no generan ajustes muy diferentes en su forma de enseñar, evaluar o planificar comparado con los docentes más jóvenes.

Para finalizar la discusión, resaltar que se evidencian diferencias entre las prácticas pedagógicas según las agrupaciones de participantes consideradas en este estudio. Las diferencias más importantes suelen hallarse en las prácticas pedagógicas inclusivas asociadas a la metodología y la evaluación. Considerando lo anterior, llama la atención que no se evidencien simultáneamente diferencias estadísticamente significativas en las prácticas asociadas a la planificación. Este hecho podría poner en manifiesto que los ajustes tanto a la evaluación como a las metodologías que declaran efectuar los docentes participantes del estudio tienden a ejecutarse sin una planificación prevista e intencionada, pudiendo ser más bien una práctica pedagógica inclusiva desde la improvisación (Jiménez et al., 2017).

\section{CONCLUSIONES}

La investigación presentada tuvo por objetivo comparar las prácticas pedagógicas inclusivas, específicamente las de planificación, metodología, evaluación, condiciones físicas del aula y uso del tiempo que los docentes de dos colegios de la Región de Coquimbo, Chile, manifiestan ejecutar. A partir de los hallazgos, se evidencia que no existe una homogeneidad en las prácticas inclusivas del profesorado. Esto deja entrever que hay aspectos que hacen variar dichas prácticas, tales como la realidad de cada establecimiento y el contexto sociocultural donde está ubicado, el tipo de estudiantado, la formación docente, y el sexo del profesorado que lleva a cabo los procesos pedagógicos.

Actualmente las aulas en Chile son muy diversas, considerando tanto las necesidades educativas formales, la matrícula de estudiantes inmigrantes en la escuela chilena, e incluso la diversidad social, cultural y económica presente en los establecimientos educacionales 
socialmente mixtos del país (Rojas, 2018). Sumado, a que el funcionamiento de los sistemas educativos es dinámico: cada colegio y cada aula es única, asimismo los docentes, los estudiantes, los diferentes agentes educativos y las propias situaciones de enseñanza y aprendizaje. Además, específicamente a nivel docente existen otros factores que pueden afectar la magnitud de las prácticas pedagógicas inclusivas que se implementen, tales como las actitudes docentes hacia la inclusión educativa (Saloviita \& Schaffus, 2016; Yada \& Savolainen, 2017) y la preocupación o sobrecarga que los docentes prevén (Malinen et al., 2013; Sharma, Aiello, Pace, Round \& Subban, 2017) a partir de la existencia de estudiantes diversos en el aula de clases.

Como se revisó en la literatura considerada en esta investigación, existen estudios previos que abordan la temática, y algunas entregan evidencia respecto de las prácticas inclusivas comparadas entre tipos de docentes según diferentes criterios de agrupación. Sin embargo, en este estudio no se contribuyó solamente con evidencia sobre el total de prácticas pedagógicas como en los estudios citados, sino que además se efectuaron estas comparaciones, diferenciando sobre cinco tipos de prácticas pedagógicas inclusivas existentes que los docentes dicen emplear en aula, y por tanto entrega evidencia más específica al respecto.

Por otra parte, en este estudio también se encuentra la limitante relacionada con el origen de la información, pues proviene desde lo que los docentes informan, como en otras investigaciones existentes en la literatura. Algunos estudios previos señalan que los docentes en Chile y países latinos suelen sobrevalorar sus prácticas pedagógicas y que por lo mismo, tienen menos autocrítica respecto de estas (Figueroa \& Muñoz, 2014; García et al., 2015), lo cual puede ser un sesgo hasta cierto nivel en este estudio. Al contrario, en países como España, Japón y Sudáfrica los docentes suelen manifestar la falta de herramientas para dar una respuesta educativa apropiada a la diversidad de alumnado (Daetxerre et al., 2013; Engelbrecht et al., 2017; González et al., 2016; Yada \& Savolainen, 2017). Aunque es importante el análisis de la información desde lo que los docentes declaran, sería interesante y necesario proyectar esta investigación complementando los datos obtenidos por medio del instrumento de autorreporte con datos que se puedan obtener desde su versión de observación. De este modo, sería posible determinar las consistencias y disonancias entre las prácticas que los docentes dicen efectuar y las que realmente se llevan a cabo en la sala de clases. Adicionalmente, sería interesante efectuar una intervención para el desarrollo e instalación de prácticas pedagógicas inclusivas, pues otros investigadores han evidenciado que los docentes se enriquecen con estos procesos de acompañamiento, a medida que también logran valorar sus prácticas pedagógicas de una manera más objetiva (Serrato \& García, 2014).

Para finalizar, es trascendental que los establecimientos educacionales se apropien del enfoque inclusivo y no hacer empleo de prácticas desde enfoques centrados en el déficit o las necesidades educativas (Forlin, Kawai \& Higuchi, 2015). Esto es relevante, pues desde el enfoque inclusivo o centrado en la diversidad se orientan las prácticas pedagógicas apropiadas, se genera una cultura de participación y aprendizaje activo, se intenciona el alcance de conocimientos y desarrollo de habilidades de todo el grupo, así como el trabajo en codocencia (Valle \& Connor, 2019). Asimismo, la implementación en aula de prácticas inclusivas no debe ser aislada, pues requiere ser abordada desde una perspectiva a nivel cultural e incluso con los apoyos económicos necesarios (Engelbrecht, Nel, Smit \& van Deventer, 2016). Así se priorizaría la sensibilización y formación permanente del profesorado, tan necesario en las aulas del siglo XXI (Hawkins et al., 2019). 


\section{REFERENCIAS BIBLIOGRÁFICAS}

Booth, T. \& Ainscow, M. (2000). Índice de inclusión. Guía para la evaluación y mejora de la educación inclusiva. Madrid: Consorcio Universitario para la Educación Inclusiva. Recuperado de www.ite.educacion.es/formacion/materiales/.../guia_indicadores_inclusiva_unesco.pdf

(2011). Index for Inclusion: developing learning and participation in schools (3rd ed.). Bristol: Centre for Studies in Inclusive Education (CSIE).

Bryant, D., Bryant, B. \& Smith, D. (2019). Teaching Students with Special Needs in Inclusive Classrooms. California: SAGE Publications.

Camacho, J. (2008). Asociación entre variables: Correlación no paramétrica. Acta Médica Costarricense, 50(3), 144-146. Recuperado de http://www.redalyc.org/pdf/434/43411549004.pdf

Chiner, E. (2011). Las percepciones y actitudes del profesorado hacia la inclusión del alumnado con necesidades educativas especiales como indicadores del uso de prácticas educativas inclusivas en el aula. (Tesis doctoral). Universidad de Alicante, España.

Coady, M., Harper, C. \& De Jong, E. (2016). Aiming for Equity: Preparing Mainstream Teachers for Inclusion or Inclusive Classrooms? TESOL Quarterly, 50, 340-368. doi: https://doi.org/10.1002/ tesq. 223

Darretxe, L., Goikoetxea, J. y Fernández, A. (2013). Análisis de prácticas inclusivas y exclusoras en dos centros educativos del país Vasco. Revista Electrónica Actualidades Investigativas en Educación, 13(2), 1-30. doi: https://www.doi.org/10.15517/aie.v13i2.11734

Engelbrecht, P., Nel, M., Smit, S. \& van Deventer, M. (2016). The Idealism of Education Policies and the Realities in Schools: The Implementation of Inclusive Education in South Africa. International Journal of Inclusive Education 20(5), 520-535. doi: https://doi.org/10.1080/1360 3116.2015.1095250

Engelbrecht, P., Savolainen, H., Nel, M., Koskela, T. \& Okkolin, M. (2017). Making meaning of inclusive education: Classroom practices in Finnish and South African classrooms. Compare: A Journal of Comparative and International Education, 47(5), 684-702. doi: https://doi.org/10.10 80/03057925.2016.12669277

Espinoza, L. y Valdebenito, V. (2018). Migración y educación en Chile: ¿Es suficiente la respuesta educativa en el actual contexto de diversidad cultural? Revista Opción, 34(87), 352-372. Recuperado de http://produccioncientificaluz.org/index.php/opcion/issue/view/2727

Figueroa, I. y Muñoz,Y.(2014). La guía para la inclusión educativa como herramienta de autoevaluación institucional: Reporte de una experiencia. Revista Latinoamericana de Educación Inclusiva, 8(2), 179-198. Recuperado de https://dialnet.unirioja.es/descarga/articulo/4994305.pdf

Flores, R. (2007). Representaciones de género de profesores y profesoras de matemática, y su incidencia en los resultados académicos de alumnos y alumnas. Revista Iberoamericana de Educación, (43), 103-118. Recuperado de https://dialnet.unirioja.es/ejemplar/159597

Florian, L. \& Black-Hawkins, K. (2011). Exploring Inclusive Pedagogy. British Educational Research Journal, 37(5), 813-828. doi: https://doi.org/10.1080/01411926.2010.501096

Forlin, C., Kawai, N. \& Higuchi, S. (2015). Educational reform in Japan towards inclusion: Are we training teachers for success? International Journal of Inclusive Education, 19(3), 314-331. https://doi.org/10.1080/13603116.2014.930519

García, I., Romero, S. y Escalante, L. (2011). Diseño y validación de la guía de evaluación de las prácticas inclusivas en aula (GEPIA). XI Congreso Nacional de Investigación Educativa. Congreso llevado a cabo en Universidad Autónoma de San Luis Potosí, México. Recuperado de www.comie.org.mx/congreso/memoriaelectronica/v11/docs/area_14/1390.pdf

García, I., Romero, S., Rubio, S., Jocabed, V. y Martínez A. (2015). Comparación de prácticas inclusivas de docentes de servicio de educación especial y regular en México. Revista Electrónica Actualidades Investigativas en Educación, 15(3), 1-17. doi: http://dx.doi.org/10.15517/aie. v15i3.20671 
Garzón Castro, P., Calvo Álvarez, M. I. y Orgaz Baz, M. B. (2016). Inclusión educativa: Actitudes y estrategias del profesorado. Revista Española de Discapacidad, 4(2), 25-45. Recuperado de https://www.cedd.net/redis/index.php/redis/article/view/19

González, F., Martín, E., Poy, R. y Jenaro, C. (2016). Percepciones del profesorado sobre la inclusión: Estudio preliminar. Revista Electrónica Interuniversitaria de Formación del Profesorado, 19(3), 11-24. doi: https://doi.org/10.6018/reifop.19.3.219321

Granada, M., Pomés, M. y Sanhueza A. (2013). Actitud de los profesores hacia la inclusión educativa. Papeles de Trabajo, (25), 51- 59. Recuperado de https://core.ac.uk/download/pdf/61700917.pdf

Hawkins, J., Crim, C., Ganz, J. B. \& Kennedy, K. D. (2019). The power of peers: Setting a course for 21 st century skills in inclusive classrooms. Hershey, Pennsylvania: IGI Global.

Jiménez, F., Aguilera, M., Valdés, R. y Hernández, M. (2017). Migración y escuela: Análisis documental en torno a la incorporación de inmigrantes al sistema educativo chileno. Psicoperspectivas, 16(1), 105-116. doi: https://www.doi.org/10.5027/psicoperspectivas-vol16-issue1-fulltext-940

Jorgensen, C. M., McSheehan, M. \& Sonnenmeier, R. M. (2007). Presumed competence reflected in the educational programs of students with IDD before and after the Beyond Access professional development intervention. Journal of Intellectual \& Developmental Disability, 32, 248-262. doi: www.doi.org/10.1080/13668250701704238

Kozleski, E., Artiles, A. \& Waitoller, F. (2014). Equity in Inclusive Education: A Cultural Historical Comparative Perspective. In L. Florian, (Ed.) The SAGE Handbook of Special (pp. 239-249). New York: Sage.

López Domínguez, A. y Diez Fumero, T. (2017). Aproximación de la estadística a las Ciencias Sociales: Una mirada crítica. Revista Cubana de Educación Superior, 36(2), 148-156. Recuperado de http://www.rces.uh.cu/index.php/RCES/article/view/165

Malinen, O., Savolainen, H., Engelbrecht, P., Xu, J., Nel, M. \& Tlale, D. (2013). Exploring Teacher Selfefficacy for Inclusive Practices in Three Diverse Countries. Teaching and Teacher Education, 33(7), 34-44. doi: www.doi.org/10.1016/j.tate.2013.02.004

Mellado, M., Chaucono, J., Hueche, M. y Aravena, O. (2017). Percepciones sobre la educación inclusiva del profesorado de una escuela con Programa de Integración Escolar. Revista de Educación, 41(1), 1-14. doi: www.doi.org/10.15517/revedu.v41i1.21597

Ministerio de Educación de Chile (2015). Ley nº 20.845 de inclusión escolar que regula la admisión de los y las estudiantes, elimina el financiamiento compartido y prohíbe el lucro en establecimientos educacionales que reciben aportes del estado. Publicada en Diario Oficial de Chile, 08 de junio del 2015. Chile. Recuperado de https://www.leychile.cl/Navegar?idNorma=1078172

Ministerio de Educación de Chile [MINEDUC]. (2015). Decreto No83 que aprueba criterios y orientaciones de adecuación curricular para estudiantes con necesidades educativas especiales de educación parvularia y educación básica. Publicado el 05 de febrero de 2015. Recuperado de https://www.leychile.cl/Navegar?idNorma=1074511

Morningstar, M. E., Shogren, K. A., Lee, H. \& Born, K. (2015). Preliminary lessons about supporting participation and learning in inclusive classrooms. Research and Practice for Persons with Severe Disabilities, 40(3), 192 -210. doi: www.doi.org/10.1177/1540796915594158

Rojas, M. T. (2018). Inclusión social: Miradas de los docentes y apoderados frente a la mixtura social en sus escuelas. Estudios Pedagógicos, 44(3), 217-234. Recuperado de http://revistas.uach.cl/ index.php/estped/article/view/4230/5280

Ryndak, D., Jackson, L. B. \& White, J. M. (2013). Involvement and progress in the general curriculum for students with extensive support needs: K-12 inclusive education research and implications for the future. Inclusion, 1, 28-49. doi: http://www.doi.org/10.1352/2326-6988-1.1.028

Salend, S. J., \& Duhaney, L. M. (2011). Historical and philosophical changes in the education of students with exceptionalities. Advances in Special Education, 21, 1-20. doi: www.doi. org/10.1108/S0270-4013(2011)0000021004

Saloviita, T. \& Schaffus, T. (2016). Teacher Attitudes Towards Inclusive Education in Finland 
and Brandenburg, Germany and the Issue of Extra Work. European Journal of Special Needs Education, 31, 458-471. doi: https://doi.org/10.1080/08856257.2016.1194569

Serrato, L. y García, I. (2014). Evaluación de un programa de intervención para promover prácticas docentes inclusivas. Actualidades Investigativas en Educación, 14(3), 1-25. Recuperado de www.scielo.sa.cr/pdf/aie/v14n3/a15v14n3.pdf

Sharma, U., Aiello, P., Pace, E. M., Round, P. \& Subban, P. (2017). In-service teachers' attitudes, concerns, efficacy and intentions to teach in inclusive classrooms: An international comparison of Australian and Italian teachers. European Journal of Special Needs Education, 33(3), 437446. doi: www.doi.org/10.1080/08856257.2017.1361139

Shogren, K. A., McCart, A. B., Lyon, K. J. \& Sailor, W. S. (2015). All means all: Building knowledge for inclusive schoolwide transformation. Research and Practice for Persons with Severe Disabilities, 40, 173-191. doi: https://doi.org/10.1177/1540796915586191

Specht, J., McGhie-Richmond, D., Loreman, T., Mirenda, P., Bennett, S., Gallagher, T., ... \& Cloutier, S. (2016). Teaching in inclusive classrooms: efficacy and beliefs of Canadian preservice teachers. International Journal of Inclusive Education, 20(1), 1-15. doi: www.doi.org/10.1080/1360311 6.2015 .1059501

Suriá, R. (2012). Discapacidad e integración educativa: ¿Qué opina el profesorado sobre la inclusión de estudiantes con discapacidad en sus clases? REOP-Revista Española de Orientación y Psicopedagogía, 23(3), 96-109. Recuperado de https://dialnet.unirioja.es/servlet/ articulo? codigo $=4405784$

Valle, J. W. \& Connor, D. J. (2019). Rethinking disability: A disability studies approach to inclusive practices. New York: Routledge.

Vicuña, K. (2013). Percepciones y actitudes de profesores de primero a séptimo año de Educación General Básica del Distrito Educativo 4 hacia la inclusión del alumnado con necesidades educativas especiales como indicadores de uno de prácticas educativas inclusivas en el aula (Tesis de maestría). Universidad Casa Grande, Ecuador.

Yada, A. \& Savolainen, H. (2017). Japanese in-service teachers' attitudes toward inclusive education and self-efficacy for inclusive practices. Teaching and Teacher Education, 64, 222-229. doi: https://doi.org/10.1016/j.tate.2017.02.005 
